\title{
Risk Factors for Loosening of S2 Alar Iliac Screw: Surgical Outcomes of Adult Spinal Deformity
}

\author{
Yasushi Iijima ${ }^{1}$, Toshiaki Kotani ${ }^{1}$, Tsuyoshi Sakuma ${ }^{1}$, Keita Nakayama ${ }^{1}$, \\ Tsutomu Akazawa ${ }^{2}$, Shunji Kishida ${ }^{1}$, Yuta Muramatsu ${ }^{1}$, Yu Sasaki ${ }^{1}$, Keisuke Ueno ${ }^{1}$, \\ Tomoyuki Asada ${ }^{1}$, Kosuke Sato ${ }^{1}$, Shohei Minami ${ }^{1}$, Seiji Ohtori ${ }^{3}$ \\ ${ }^{I}$ Department of Orthopedic Surgery, Seirei Sakura Citizen Hospital, Sakura, Japan \\ ${ }^{2}$ Department of Orthopedic Surgery, St. Marianna University School of Medicine, Kawasaki, Japan \\ ${ }^{3}$ Department of Orthopedic Surgery, Graduate School of Medicine, Chiba University, Chiba, Japan
}

\begin{abstract}
Study Design: Retrospective study.
Purpose: To determine the risk factors for S2 alar iliac (S2Al) screw loosening and its association with lumbosacral fusion in patients with adult spinal deformity (ASD).

Overview of Literature: S2Al screws have been widely used for ASD surgery in recent years. However, no studies have analyzed the risk factors for loosening of S2Al screws and its association with lumbosacral fusion.

Methods: Cases of 50 patients with ASD who underwent long spinal fusion (>9 levels) with S2Al screws were retrospectively reviewed. Loosening of S2Al screws and S1 pedicle screws and bone fusion at the level of L5-S1 at 2 years after surgery were investigated using computed tomography. In addition, risk factors for loosening of S2Al screws were determined in patients with ASD.

Results: At 2 years after surgery, 33 cases (66\%) of S2Al screw loosening and six cases (12\%) of S1 pedicle screw loosening were observed. In 40 of 47 cases (85\%), bone fusion at L5-S1 was found. Pseudarthrosis at L5-S1 was not significantly associated with S2Al screw loosening (19.3\% vs. $6.3 \%, p=0.23)$, but significantly higher in patients with $\mathrm{S} 1$ screw loosening $(83.3 \%$ vs. $4.9 \%$, $p<0.001$ ). On multivariate logistic regression analyses, high upper instrumented vertebra (UIV) level (T5 or above) (odds ratio [OR], 4.4; $95 \%$ confidence interval [CI], 1.0-18.6; $p=0.045)$ and obesity $(0 \mathrm{R}, 11.4 ; 95 \% \mathrm{Cl}, 1.2-107.2 ; p=0.033)$ were independent risk factors for S2Al screw loosening.

Conclusions: High UIV level (T5 or above) and obesity were independent risk factors for S2Al screw loosening in patients with lumbosacral fixation in surgery for ASD. The incidence of lumbosacral fusion is associated with S1 screw loosening, but not S2Al screw loosening.
\end{abstract}

Keywords: Adult spinal deformity; Lumbosacral fusion; Lumbosacral region; S2 alar iliac screw; Screw loosening

\section{Introduction}

Lumbosacral fixation is crucial in maintaining sagittal and coronal balance and achieving bone fusion in adult spinal deformity (ASD) surgery. However, S1 pedicle screws without iliac fixation were associated with a high

Received Mar 10, 2020; Revised Apr 21, 2020; Accepted Apr 22, 2020

Corresponding author: Yasushi Iijima

Department of Orthopedic Surgery, Seirei Sakura Citizen Hospital, 2-36-2 Ebaradai, Sakura-city, Chiba, 285-8765, Japan

Tel: +81-43-486-1151, Fax: +81-43-486-8696, E-mail: yi02008@yahoo.co.jp 
rate of pseudarthrosis at the lumbosacral joint (33\%) and implant failure (44\%) [1,2]. Sacropelvic fixation by adding iliac screws distal to the $\mathrm{S} 1$ pedicle screws may decrease the force on the S1 pedicle screws and improve lumbosacral fusion [3]. S2 alar iliac (S2AI) screws may achieve less revision surgery with lower-profile and lower-rate loosening compared with iliac screws as an anchor of sacropelvic fixation [4-7].

Screw loosening is represented by a radiolucent area around the screw and indicates loss of screw fixation and may cause pseudarthrosis [8]. Risk factors for loosening of $\mathrm{S} 1$ pedicle screws and iliac screw have been investigated $[9,10]$. Some studies found a possible etiology of S2AI screw loosening $[11,12]$, that is, S2AI screws penetrating the sacroiliac joint without bone fusion of the joint. However, apparently no studies have analyzed the risk factors for S2AI screw loosening. In addition, to our knowledge, the association of S2AI screw loosening with lumbosacral fusion has not yet been reported.

This study aimed to determine risk factors for S2AI screw loosening and its association with lumbosacral fusion in patients with ASD.

\section{Materials and Methods}

\section{Patient population}

Records of 55 consecutive patients with ASD who underwent long instrumented spine fusion ( $>9$ levels) using S2AI screws in Seirei Sakura Citizen Hospita from November 2011 to April 2016 were retrospectively reviewed. All patients had a minimum of 2-year follow-up. Patients who underwent whole spine anteroposterior and lateral standing radiography and computed tomography (CT) preoperatively, immediately postoperatively, and 2 years postoperatively were included. Five patients who lacked postoperative CT were excluded. Thus, this present study included cases of 50 patients. This study protocol, including review of patient records, was approved by the review board of Seirei Sakura Citizen Hospital (IRB approval no., 30-12). Informed consent was obtained from all patients included in the study.

\section{Surgical procedure}

All S2AI screws had been inserted using an O-armassisted spinal navigation system (Medtronic Sofamor
Danek, Memphis, TN, USA). S2AI screws with diameter of 7.5 or $8.5 \mathrm{~mm}$ and length of $50-90 \mathrm{~mm}$ were used. Deformity correction had been performed in all patients via vertebral column resection (VCR), pedicle subtraction osteotomy (PSO), or lateral lumber interbody fusion with a cancellous allograft and autograft. The number of levels fused and correction techniques were at the discretion of the attending surgeons. Transforaminal lumbar interbody fusion had been usually performed at L5-S1 using a polyetheretherketone cage with a cancellous allograft except for cases of preoperative spontaneous bone bridging at L5-S1. Th10 were selected for UIV, but Th5 or higher for patients with thoracic scoliosis and Parkinson's disease. All surgery had been performed by the same team of four experienced spine surgeons.

\section{Patient data}

Baseline characteristics were retrospectively investigated, including age at surgery, sex, body mass index (BMI, kg/ $\mathrm{m}^{2}$ ), bone mineral density (BMD), instrumented fusion levels, upper instrumented vertebra (UIV) level (T5 and above or below T5), with or without L5-S1 interbody fusion, and diameter and length of S2AI screws. BMD of lumbar spine was influenced by degeneration and did not exactly reflect osteoporosis; hence, BMD of the femoral neck had been determined by dual-energy X-ray absorptiometry.

\section{Radiographic analysis}

In the lateral whole spine radiographs, sagittal vertical axis (SVA) was measured as the distance from the posterosuperior edge of the S1 to the C7 plumb line. Pelvic incidence (PI) was measured from the angle subtended by a perpendicular from the cephalad endplate of $\mathrm{S} 1$ and a line connecting the center of the femoral head to the center of the cephalad endplate of S1. Thoracic kyphosis (TK) was measured as the angle from the upper endplate of T5 to the lower endplate of T12, and lumbar lordosis (LL) was measured as the angle from the upper endplate of L1 to the upper endplate of the S1. PI-LL was obtained as global spine alignment.

On CT images obtained 2 years postoperatively, signs of loosening of the S1 pedicle screws and S2AI screws on both right and left sides were examined. Loosening was defined as a radiolucent area $(>1.5 \mathrm{~mm})$ around the screw 


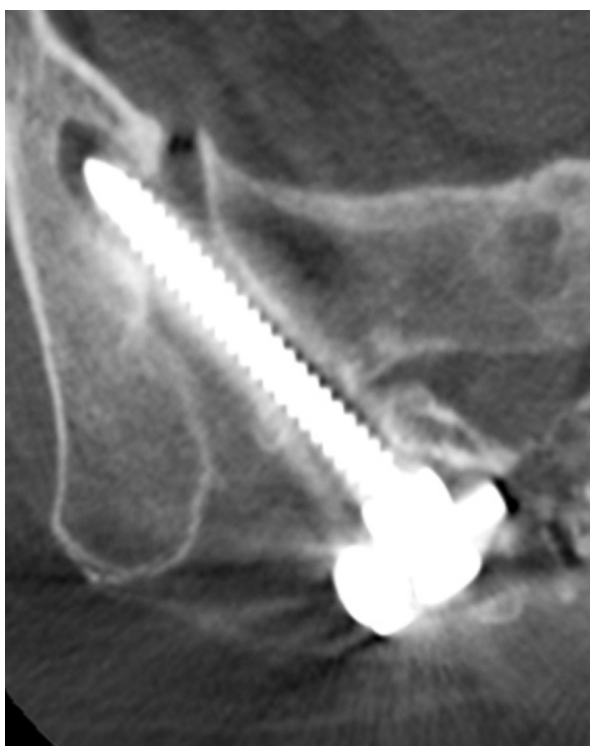

Fig. 1. Representative sacral and pelvic multiplanar reconstruction CT images of a patient with adult spinal deformity after surgery. CT shows a radiolucent zone around the screw. Loosening was defined as a radiolucent zone $>1.5 \mathrm{~mm}$. CT, computed tomography.

Table 1. Kappa coefficients for intraobserver and interobserver reliability

\begin{tabular}{lcc} 
& Intraobserver & Interobserver \\
S1 screw loosening & 0.79 & 0.66 \\
S2Al screw loosening & 0.95 & 0.95 \\
Bone fusion at L5/S1 & 1.0 & 1.0 \\
\hline
\end{tabular}

S2Al screw, S2 alar iliac screw.

(Fig. 1) [9]. Bone fusion at L5-S1 in CT images obtained 2 years postoperatively was examined. Bone fusion was defined as a bridging bone formation in the L5-S1 disk space, the L5-S1 facet joint, or between the L5 transverse process and S1 alar $[13,14]$.

To evaluate intraobserver and interobserver reliability, 20 of 50 cases were randomly selected. Loosening of S1 and S2AI screws and bone fusion at the L5-S1 were assessed from CT images by two orthopedic spinal surgeons who were both blinded to clinical data and outcome. The observer reviewed the same patient series in a second session. Kappa coefficients were used to evaluate the reproducibility of loosening and bone fusion judgment [15]. The intraobserver and interobserver reliabilities were acceptable (Table 1).

\section{Statistical analysis}

Student $t$-test was used to analyze continuous variables,
Table 2. Patient and instrument characteristics

\begin{tabular}{|c|c|}
\hline Characteristic & Value \\
\hline \multicolumn{2}{|l|}{ Patient characteristics } \\
\hline Total no. of patients & 50 \\
\hline Age at the time of surgery (yr) & $67.3 \pm 8.0$ \\
\hline Sex (male vs. female) & $5: 45$ \\
\hline Bone mineral density $\left(\mathrm{g} / \mathrm{cm}^{2}\right)$ & $0.59 \pm 0.1$ \\
\hline Bone mass index $\left(\mathrm{kg} / \mathrm{m}^{2}\right)$ & $23.4 \pm 4.5$ \\
\hline Obesity (no. of patients) & 14 \\
\hline \multicolumn{2}{|l|}{ Instrument characteristics } \\
\hline Instrumented fusion levels & $12.0 \pm 2.7$ \\
\hline $\begin{array}{l}\text { Upper instrumented vertebra ( } \mathrm{T} 5 \text { or above vs. below } \\
\text { T5) }\end{array}$ & $26: 24$ \\
\hline L5/S interbody fusion (yes vs. no) & $40: 10$ \\
\hline Screw diameter ( $\varphi 7.5 \mathrm{~mm}$ vs. $\varphi 8.5 \mathrm{~mm}$ ) & $9: 41$ \\
\hline Screw length (mm) & $72.2 \pm 13.0$ \\
\hline
\end{tabular}

and Fisher's exact test for categorical variables. Patients were divided into two groups according to the presence of S2AI screw loosening (loosening group [at least one loose S2AI screw] and a nonloosening group). Age, sex, BMI, BMD, instrumented fusion levels, UIV level, with or without L5-S1 interbody fusion, diameter and length of S2AI screws, and spinopelvic parameters (SVA, PI, TK, LL, PI-LL) were compared between the two groups. A multivariate logistic regression analysis was conducted to identify predictors of S2AI screw loosening. Obesity, UIV level, preoperative SVA, and PI-LL were included in the multivariate model, because these factors had a $p<0.25$ in the univariate analyses and were considered clinically important. Odds ratios (ORs) were reported with 95\% confidence intervals (CIs). All $p<0.05$ were considered statistically significant. JMP software package ver. 10.0 (SAS Institute Inc., Cary, NC, USA) was used for all analyses.

\section{Results}

Patient characteristics and instrument data for spinal fusion are presented in Table 2 . The mean age at the time of surgery was $67.3 \pm 8.0$ years (range, $47-80$ years). There were 45 female and five male patients. Their average BMI was $23.4 \pm 4.5 \mathrm{~kg} / \mathrm{m}^{2}$ (range, $18.0-40.1 \mathrm{~kg} / \mathrm{m}^{2}$ ). Fourteen patients $(28 \%)$ whose BMI was $\geq 25 \mathrm{~kg} / \mathrm{m}^{2}$ were considered obese. The average of BMD was $0.59 \pm 0.1 \mathrm{~g} / \mathrm{cm}^{2}$ (range, $0.31-0.74 \mathrm{~g} / \mathrm{cm}^{2}$ ). Twenty-one patients had poste- 

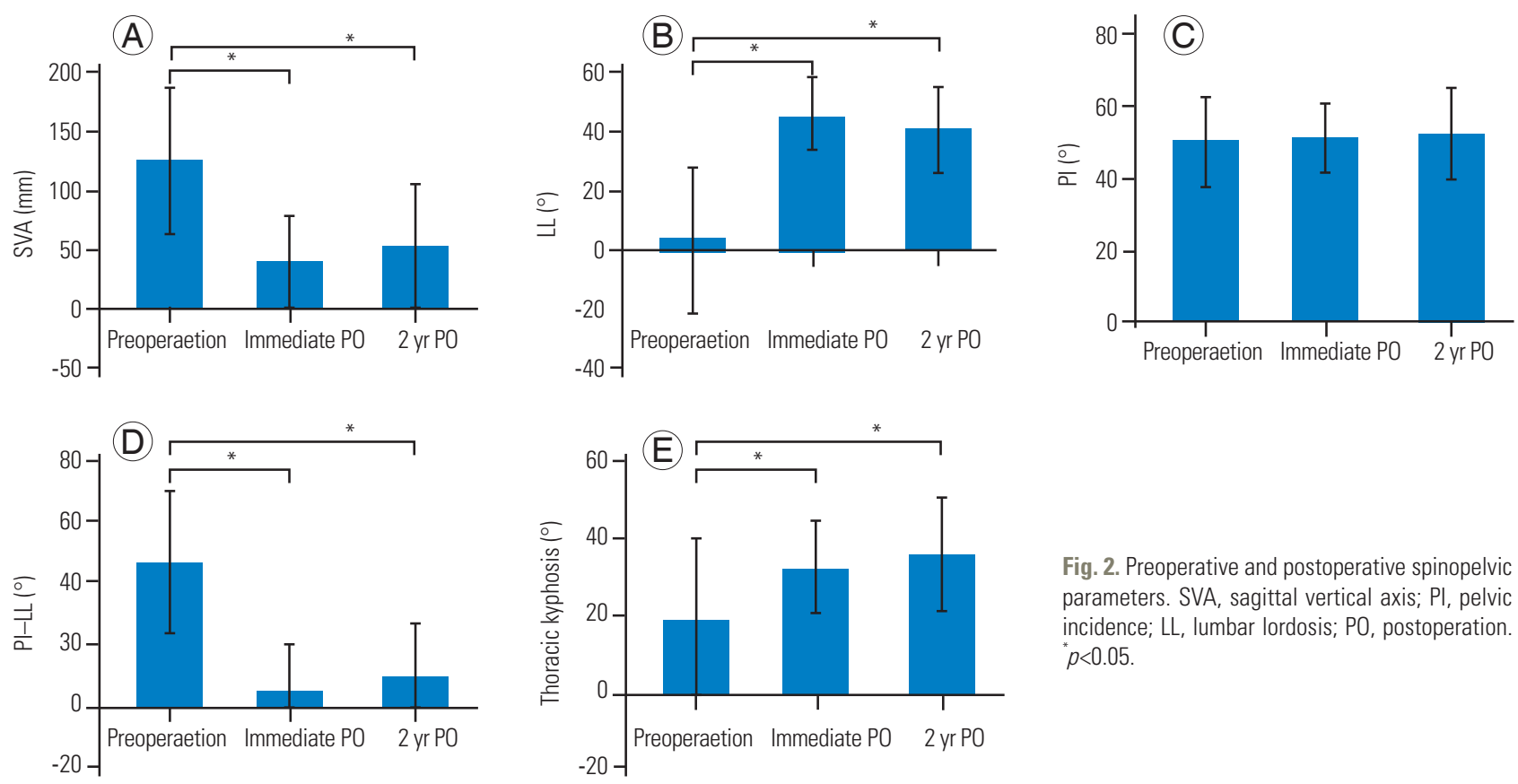

Fig. 2. Preoperative and postoperative spinopelvic parameters. SVA, sagittal vertical axis; PI, pelvic incidence; LL, lumbar lordosis; PO, postoperation. " $p<0.05$.

rior only instrumented fusion, and 29 patients had combined anterior and posterior instrumented spinal fusion. The average number of instrumented fusion levels was 12.0 2.7 (range, 9-16). Twenty-six patients had UIV at T5 or above, and 24 patients below T5. An interbody fusion at L5-S1 had been performed in 40 patients and was not performed in 10 patients (three patients had preoperative spontaneous bone bridging at L5-S1). S2AI screws with 7.5-mm diameter were used in nine patients, and screws with $8.5-\mathrm{mm}$ diameter were used in 41 patients. The average length of the S2AI screws was $72.2 \pm 13.0 \mathrm{~mm}$ (range, 40-90 mm).

\section{Surgical outcomes}

Preoperative and postoperative spinopelvic parameters are presented in Fig. 2. Preoperative, immediately postoperative, and 2 years postoperative SVA was $127.6 \pm 62.8$ $\mathrm{mm}, 39.2 \pm 40.7 \mathrm{~mm}$, and $53.3 \pm 51.9 \mathrm{~mm}$, respectively. Preoperative, immediately postoperative, and 2 years postoperative PI-LL was $46.2^{\circ} \pm 21.4^{\circ}, 4.9^{\circ} \pm 15.0^{\circ}$, and $9.9^{\circ} \pm 16.7^{\circ}$, respectively. Spinopelvic parameters improved soon after surgery and were maintained during the 2-year follow-up period. Bone fusion at L5-S1 was found in 40 of $47 \mathrm{pa}-$ tients $(85 \%)$ except for three with spontaneous preoperative bone fusion.
Table 3. Relationship between S2Al screw and S1 PS loosening

\begin{tabular}{lccc}
\multirow{2}{*}{ S1 PS loosening } & \multicolumn{2}{c}{ S2Al screw loosening } & \\
\cline { 2 - 3 } & Yes $(n=33)$ & No $(n=17)$ & \\
Yes & 6 & 0 & 0.061 \\
No & 27 & 17 & \\
\hline
\end{tabular}

Three cases were excluded in this analysis because of preoperative spontaneous fusion.

S2Al screw, S2 alar iliac screw; PS, pedicle screw.

\section{S2 alar iliac screw loosening}

Table 3 shows the relationship between S2AI and S1 pedicle screw loosening. S2AI screw loosening was found in 33 patients (66\%): unilateral in 17 and bilateral in 16 . S1 pedicle screw loosening was found in six patients (12\%): unilateral in two and bilateral in four. To investigate the association between S2AI and S1 pedicle screw loosening, the screw was judged as loose if a screw on one of the sides was loose. All cases with S1 pedicle screw loosening also showed S2AI screw loosening. However, the S1 pedicle screw loosening rate was not significantly different between the S2AI screw loosening group and nonloosening group ( $18.2 \%$ versus $0 \%, p=0.061$ ).

Using O-arm navigation, all S2AI screws properly penetrated the sacroiliac joint, and there was no S2AI or S1 screw misplacement. Revision surgery was required in 
two cases in the S2AI screw loosening group due to L5-S pseudarthrosis with S2AI screw breakage and rod breakage at L5-S1. In addition, one patient in each group had proximal junctional failure requiring reoperation, and three patients (two in the S2AI screw loosening group and one in the S2AI screw nonloosening group) had rod breakage at the level of PSO or VCR requiring reoperation.

Pseudarthrosis rate at the level of L5-S1 was not significantly associated with S2AI screw loosening (19.3\% versus $6.3 \%, p=0.23$ ) (Table 4 ), but was significantly higher in patients with S1 screw loosening (83.3\% versus $4.9 \%$, $p<0.001$ ) (Table 5).

\section{Risk factors}

Significant differences were found in obesity, preoperative PI, and postoperative PI-LL, PI, and SVA between S2AI

Table 4. Relationship between S2AI screw loosening and bone fusion at L5-S1

\begin{tabular}{|c|c|c|c|}
\hline \multirow{2}{*}{ Bone fusion at L5-S1 } & \multicolumn{2}{|c|}{ S2Al screw loosening } & \multirow{2}{*}{$p$-value } \\
\hline & Yes $(n=31)$ & No $(n=16)$ & \\
\hline Yes & 25 & 15 & 0.23 \\
\hline No & 6 & 1 & \\
\hline
\end{tabular}

Three cases were excluded in this analysis because of preoperative spontaneous fusion.

S2Al screw, S2 alar iliac screw.

Table 5. Relationship between S1 screw loosening and bone fusion at L5-S1

\begin{tabular}{|c|c|c|c|}
\hline \multirow{2}{*}{ Bone fusion at L5-S1 } & \multicolumn{2}{|c|}{ S1 screw loosening } & \multirow{2}{*}{$p$-value } \\
\hline & Yes $(n=6)$ & No $(n=41)$ & \\
\hline Yes & 1 & 39 & $<0.001^{*}$ \\
\hline No & 5 & 2 & \\
\hline
\end{tabular}

Three cases were excluded in this analysis because of preoperative spontaneous fusion.

*Statistically significant.

screw loosening and nonloosening groups $(p<0.05)$ (Table 6). On multivariate logistic regression analyses with four potential predictors (obesity, UIV level, preoperative SVA, and preoperative PI-LL), high UIV level (T5 or above) (OR, 4.4; 95\% CI, 1.0-18.6; $p=0.045$ ) and obesity (OR,

Table 6. Comparison between S2AI screw loosening and non-loosening group

\begin{tabular}{|c|c|c|c|}
\hline \multirow{2}{*}{ Variable } & \multicolumn{2}{|c|}{ S2Al screw loosening } & \multirow{2}{*}{$p$-value } \\
\hline & Yes $(n=33)$ & No $(n=17)$ & \\
\hline Age (yr) & $67.2 \pm 8.9$ & $67.8 \pm 6.0$ & 0.920 \\
\hline Sex (male vs. female) & $4: 29$ & $1: 16$ & 0.48 \\
\hline Obesitya) (no. of patients) & 13 & 1 & $0.012^{*}$ \\
\hline Bone mineral density $\left(\mathrm{g} / \mathrm{cm}^{2}\right)$ & $0.59 \pm 0.11$ & $0.61 \pm 0.08$ & 0.6 \\
\hline $\begin{array}{l}\text { Upper instrumented vertebra } \\
\text { (T5 or above vs. below T5) }\end{array}$ & $19: 14$ & $5: 12$ & 0.059 \\
\hline L5/S interbody fusion (yes vs. no) & $26: 7$ & $14: 3$ & 0.77 \\
\hline $\begin{array}{l}\text { Screw diameter } \\
\text { ( } \varphi 7.5 \mathrm{~mm} \text { vs. } \varphi 8.5 \mathrm{~mm})\end{array}$ & $8: 25$ & $1: 16$ & 0.11 \\
\hline Screw length (mm) & $70.6 \pm 14.1$ & $75.6 \pm 10.1$ & 0.23 \\
\hline \multicolumn{4}{|l|}{ Sagittal vertical axis (mm) } \\
\hline Preoperation & $140.0 \pm 64.0$ & $102.1 \pm 54.3$ & 0.050 \\
\hline Immediate postoperation & $48.2 \pm 42.8$ & $18.2 \pm 30.3$ & $0.028^{*}$ \\
\hline \multicolumn{4}{|l|}{$\operatorname{LL}\left({ }^{\circ}\right)$} \\
\hline Preoperation & $3.5 \pm 26.3$ & $6.7 \pm 23.3$ & 0.80 \\
\hline Immediate postoperation & $44.8 \pm 13.6$ & $49.8 \pm 8.3$ & 0.26 \\
\hline \multicolumn{4}{|l|}{$\operatorname{PI}\left({ }^{\circ}\right)$} \\
\hline Preoperation & $53.5 \pm 13.6$ & $44.8 \pm 7.6$ & $0.014^{*}$ \\
\hline Immediate postoperation & $53.6 \pm 10.5$ & $46.5 \pm 5.2$ & $0.0079 *$ \\
\hline \multicolumn{4}{|l|}{ Thoracic kyphosis $\left({ }^{\circ}\right)$} \\
\hline Preoperation & $20.2 \pm 20.6$ & $16.1 \pm 20.3$ & 0.45 \\
\hline Immediate postoperation & $31.7 \pm 12.0$ & $32.6 \pm 11.6$ & 0.77 \\
\hline \multicolumn{4}{|l|}{$\mathrm{PI}-\mathrm{LL}\left({ }^{\circ}\right)$} \\
\hline Preoperation & $50.0 \pm 21.0$ & $38.1 \pm 20.9$ & 0.084 \\
\hline Immediate postoperation & $8.8 \pm 16.0$ & $-3.3 \pm 9.1$ & $0.0079 *$ \\
\hline
\end{tabular}

Table 7. Logistic regression analysis of risk factors for S2 alar iliac screw loosening

\begin{tabular}{lcc} 
& $p$-value & Odds ratio (95\% confidence interval) \\
\hline Obesity (body mass index $\geq 25 \mathrm{~kg} / \mathrm{m}^{2}$ ) & $0.033^{*}$ & $11.4(1.2-107.2)$ \\
Upper instrumented vertebra T5 or above & $0.045^{*}$ & $4.4(1.0-18.6)$ \\
\hline Preoperative sagittal vertical axis & 0.66 & 0.37 \\
Preoperative pelvic incidence-lumbar lordosis & & \\
\hline
\end{tabular}

"Statistically significant. 

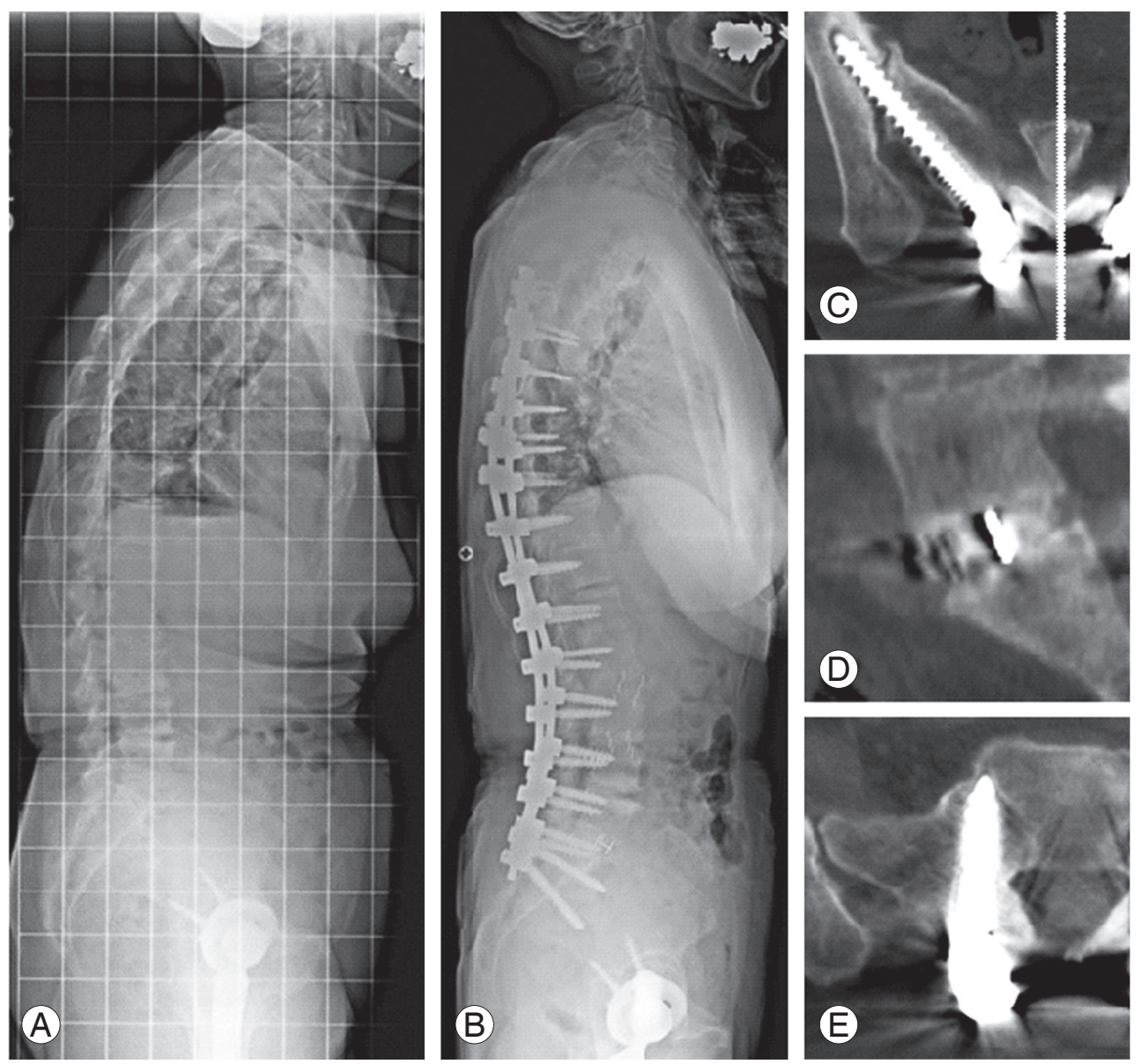

Fig. 3. A 68-year-old obese female case. Preoperative (A) and postoperative (B) whole spine lateral radiographs. Two-year postoperative multiplanar reconstruction computed tomography showed bony fusion was confirmed at the L5/S1 (D) without S1 screw loosening (E), despite S2 alar iliac bilateral screw loosening (C).

11.4; 95\% CI, 1.2-107.2; $p=0.033$ ) were independent risk factors of S2AI screw loosening (Table 7).

The representative obese case of S2AI screw loosening with high UIV level was presented (Fig. 3). This patient was a 68 -year-old obese woman (BMI, $\left.25.1 \mathrm{~kg} / \mathrm{m}^{2}\right)$. Deformity correction was performed by lateral lumbar interbody fusion and $\mathrm{T} 5$ to pelvic posterior fixation. Two-year postoperative multiplanar reconstruction $\mathrm{CT}$ showed that bony fusion was confirmed at the L5-S1 without S1 screw loosening, despite S2AI bilateral screw loosening.

\section{Discussion}

High UIV level (T5 or above) and obesity were found to be independent risk factors for S2AI screw loosening after lumbosacral fixation in patients with ASD. The incidence of lumbosacral fusion was associated with S1 screw loosening, but not S2AI screw loosening. To our knowledge, this is the first study to reveal risk factors for S2AI screw loosening and the association between lumbosacral bone fusion and loosening of S1 and S2AI screws.

A high UIV level (T5 or above) was found to be a risk factor for S2AI screw loosening after lumbosacral fixation in patients with ASD. Orita et al. [9] reported that longer fusion using S1 screws in lumbosacral fixation including the lumbosacral junction to treat spondylolisthesis increased the incidence of radiographically defined loosening, and suggested that this was because of the increased local loading to the sacrum [9]. In this study, we found that the risk factors for S2AI screw loosening associated with a high UIV level were consistent with the study by Orita et al. [9]. The long lever arm of the constructs was determined to induce a large cantilever force and provide an explanation for the association.

Furthermore, obesity was found as a risk factor for S2AI screw loosening after lumbosacral fixation in patients 
with ASD. To our knowledge, there are no previous reports revealing the association between obesity and the loosening. High BMI was a risk factor for correction loss after surgery to correct ASD [10]. Anterior translation of the trunk due to a high BMI would increase the magnitude of mechanical stress on the posterior instrument postoperatively. The mechanical stress caused by obesity may increase screw loosening $[1,9]$.

S2AI screw loosening was found in $67 \%$ of patients, and S1 screw loosening in $12 \%$ of patients 2 years after ASD surgery. Previous studies found that S2AI screw loosening was found in $2.9 \%-10.4 \%[5,7,11]$. The incidence of S2AI screw loosening found in our study was more than that reported previously. The difference was because we evaluated screw loosening using CT, while previous studies used conventional radiographs $[7,11]$. We consider that our 2-year postoperative evaluation using $\mathrm{CT}$ is more accurate than that of previous studies.

We also found pseudarthrosis at the level of L5-S1 in about $15 \%$ of patients 2 years postoperatively. Previous studies found pseudarthrosis at L5-S1 in long fusion cases using S2AI screws in $4.3 \%-6.1 \%$ of patients $[6,11]$. The incidence of pseudarthrosis found in this study is greater than that reported previously. The difference was because we evaluated pseudarthrosis using CT in all cases, while previous studies used conventional radiographs. In addition, this study showed that the incidence of pseudarthrosis was not different whether or not there was S2AI screw loosening. The incidence of pseudarthrosis was greater in patients with S1 screw loosening. The incidence of S1 pedicle screw loosening (12\%) was relatively rare compared with S2AI screw loosening (66\%). These findings were because lumbopelvic fixation protects the $S 1$ pedicle screws and improves the chance of successful lumbosacral fusion $[13,16]$. Even if a S2AI screw was loosened, lumbosacral fusion could still be obtained as long as S1 pedicle screws were not also loosened.

Our findings indicate that patients with a high UIV level or obesity should be observed carefully with strict brace protection. Moreover, patients with ASD and these risk factors should be taught not to move their lumbosacral spine violently until bony fusion is established to prevent S2AI screw loosening.

This study has several limitations. First, clinical outcome scores such as the Oswestry Disability Index and Scoliosis Research Society-22 Questionnaire were not analyzed, because we considered these questionnaires lack- ing information specifically related to pelvic fixation and we could not compare clinical outcomes related to S2AI screw loosening. Second, the risk factors for S1 screw loosening were not analyzed because the sample size for S1 screw loosening was too small (only six cases) to determine risks. In addition, the association between $\mathrm{S} 1$ and S2AI screw loosening was almost significant $(p<0.061)$, which may be due to the smaller sample size. Third, the detailed pathophysiology of ASD in our patients was heterogeneous. Patients ranged from those with primary de novo scoliosis and progressive idiopathic scoliosis to deformity secondary to osteoporotic vertebral fracture. Due to a small sample size, risk factors could not be analyzed according to patient pathophysiology. Despite these limitations, our results provide important information for surgical treatment of ASD using S2AI screws.

\section{Conclusions}

This study showed a high UIV level (T5 or above) and obesity were independent risk factors for S2AI screw loosening after lumbosacral fixation in patients with ASD. We also found that the incidence of lumbosacral fusion was associated with S1 screw loosening, but not S2AI screw loosening. Patients with a high UIV level or obesity should be observed carefully until lumbosacral bony fusion.

\section{Conflict of Interest}

No potential conflict of interest relevant to this article was reported.

\section{Acknowledgments}

We thank Ms. Yuri Ichikawa for collecting data.

\section{References}

1. Camp JF, Caudle R, Ashmun RD, Roach J. Immediate complications of Cotrel-Dubousset instrumentation to the sacro-pelvis: a clinical and biomechanical study. Spine (Phila Pa 1976) 1990;15:932-41.

2. Devlin VJ, Boachie-Adjei O, Bradford DS, Ogilvie JW, Transfeldt EE. Treatment of adult spinal deformity with fusion to the sacrum using CD instrumentation. J Spinal Disord 1991;4:1-14. 
3. Kuklo TR, Bridwell KH, Lewis SJ, et al. Minimum 2-year analysis of sacropelvic fixation and L5-S1 fusion using S1 and iliac screws. Spine (Phila Pa 1976) 2001;26:1976-83.

4. O’Brien JR, Yu WD, Bhatnagar R, Sponseller P, Kebaish KM. An anatomic study of the S2 iliac technique for lumbopelvic screw placement. Spine (Phila Pa 1976) 2009;34:E439-42.

5. Ishida W, Elder BD, Holmes C, et al. S2-alar-iliac screws are associated with lower rate of symptomatic screw prominence than iliac screws: radiographic analysis of minimal distance from screw head to skin. World Neurosurg 2016;93:253-60.

6. Ishida W, Elder BD, Holmes C, et al. Comparison between S2-alar-iliac screw fixation and iliac screw fixation in adult deformity surgery: reoperation rates and spinopelvic parameters. Global Spine J 2017;7:67280.

7. Elder BD, Ishida W, Lo SL, et al. Use of S2-alar-iliac screws associated with less complications than iliac screws in adult lumbosacropelvic fixation. Spine (Phila Pa 1976) 2017;42:E142-9.

8. Tokuhashi Y, Matsuzaki H, Oda H, Uei H. Clinical course and significance of the clear zone around the pedicle screws in the lumbar degenerative disease. Spine (Phila Pa 1976) 2008;33:903-8.

9. Orita S, Ohtori S, Eguchi Y, et al. Radiographic evaluation of monocortical versus tricortical purchase approaches in lumbosacral fixation with sacral pedicle screws: a prospective study of ninety consecutive patients. Spine (Phila Pa 1976) 2010;35:E1230-7.
10. Banno T, Hasegawa T, Yamato Y, et al. Prevalence and risk factors of iliac screw loosening after adult spinal deformity surgery. Spine (Phila Pa 1976) 2017;42:E1024-30.

11. Smith EJ, Kyhos J, Dolitsky R, Yu W, O’Brien J. S2 alar iliac fixation in long segment constructs, a twoto five-year follow-up. Spine Deform 2018;6:72-8.

12. Ilyas H, Place H, Puryear A. A comparison of early clinical and radiographic complications of iliac screw fixation versus S2 alar iliac (S2AI) fixation in the adult and pediatric populations. J Spinal Disord Tech 2015;28:E199-205.

13. Mazur MD, Mahan MA, Shah LM, Dailey AT. Fate of S2-alar-iliac screws after 12 -month minimum radiographic follow-up: preliminary results. Neurosurgery 2017;80:67-72.

14. Ohtori S, Inoue G, Orita S, et al. Teriparatide accelerates lumbar posterolateral fusion in women with postmenopausal osteoporosis: prospective study. Spine (Phila Pa 1976) 2012;37:E1464-8.

15. Sakai Y, Takenaka S, Matsuo Y, et al. Hounsfield unit of screw trajectory as a predictor of pedicle screw loosening after single level lumbar interbody fusion. J Orthop Sci 2018;23:734-8.

16. Sutterlin CE 3rd, Field A, Ferrara LA, Freeman AL, Phan K. Range of motion, sacral screw and rod strain in long posterior spinal constructs: a biomechanical comparison between S2 alar iliac screws with traditional fixation strategies. J Spine Surg 2016;2:266-76. 